\title{
On the Notion of Equivalent Specific Liquid Water Content and Its Applications to the Thermodynamics of Clouds
}

\author{
K. E. HAMAN ${ }^{1}$ \\ National Center for Almospheric Research ${ }^{2}$, Boulder, Colorado 80302
}

(Manuscript received 24 August 1972, in revised form 22 June 1973)

\begin{abstract}
A linear combination of specific liquid water content and temperature excess over the environmental value is found, which for an entrained, nonprecipitating parcel has the property of being approximately independent of environmental humidity. The proofs of this and some other properties are given and certain applications proposed.
\end{abstract}

\section{Introduction}

One of the most important problems in the analysis of thermodynamic processes in convective clouds is the determination of the temperature excess over the environmental value and the liquid water content, particularly when entrainment of environmental air occurs. In the latter case, determination, or at least estimation, of the coefficient of entrainment often appears desirable. However, all of these quantities are interconnected with each other and with external stratification of temperature and humidity in a rather complicated way. Thus, parameters of clear physical significance related to those quantities and to parameters easily available by routine measurements may be of considerable value in cloud studies.

In this paper, the following quantity, $W$, is proposed:

$$
W=V-\frac{c_{p}}{L}\left(T-T_{0}\right)
$$

where $V$ is the specific liquid water content (mass of liquid water per unit mass of "wet" air), $T$ the cloud temperature, $T_{0}$ the environmental temperature at the same pressure, $c_{p}$ the specific heat of air at constant pressure, and $L$ the latent heat of condensation. The quantity $W$ is approximately equal to the specific liquid water content that would be found in the cloud parcel, after the parcel's temperature had become equal to that of the environment through adiabatic evaporation or condensation of part of its water content. Because of a formal resemblance to the concept of equivalent temperature, it is proposed to call $W$ the equivalent specific liquid water content (ESLWC).

${ }^{1}$ On leave from the Institute of Geophysics, University of Warsaw, Poland.

${ }^{2}$ The National Center for Atmospheric Research is sponsored by the National Science Foundation.
It can be shown (see Appendix) that for a cloud parcel which isobarically entrains unsaturated environmental air but does not exchange mass, heat or water substance in any other way, changes of the ESLWC with pressure are described with relatively high accuracy by

$$
\frac{d W}{d p}=\frac{c_{p}}{L}\left(\gamma-\Gamma_{d}\right)-\mu W
$$

where $p$ is the pressure ${ }^{3} ; \gamma=d T_{0} / d p ; \Gamma_{d}$ (the dry adiabatic lapse rate with respect to pressure) $=v / c_{p}$ ( $v$ being the specific volume of the air); and $\mu$ (the coefficient of entrainment) $=d(\ln M) / d p$ ( $M$ being the mass of the parcel). If $\mu \equiv 0$, the parcel undergoes an adiabatic process. The ESLWC resulting in such a process (with given initial value) will be called the adiabatic ESLWC.

The main advantage of the ESLWC is that to the extent that the influence of entrainment on the value of $\Gamma_{d}$ can be disregarded (in practice nearly always), its changes with pressure described by Eq. (2) are independent of external humidity and depend only on entrainment and external temperature.

Furthermore, with the use of Eq. (2) the two following important theorems for the ESLWC can be demonstrated:

Theorem 1. If $\gamma<\Gamma_{d}$, and the ESLWC of the parcel is nonnegative at the cloud base--both conditions are likely to be fulfilled in typical convective conditions - -

\footnotetext{
${ }^{3}$ The choice of pressure as the independent variable (rather than the apparently more natural choice, height) is justified because it permits the expression of the properties of the ESLWC in purely thermodynamical terms, without additional discussion of the pressure/temperature/height interrelations. If, in particular applications, other independent variables such as time, height, or path length are more convenient, they can easily be introduced by suitable parameterization of pressure.
} 
the ESLWC will remain nonnegative along the parcel's entire path, provided there are no downward motions caused by nonbuoyant forces.

Theorem 2. Under the same conditions and restrictions as for Theorem 1 the ESLWC of the parcel cannot surpass the adiabatic value, provided the dependence of $\Gamma_{d}$ on entrainment can be disregarded.

Theorem 1 for the upward branch of the parcel's trajectory follows from the fact that for $\gamma<\Gamma_{d}$ the free term of Eq. (2) is negative. This means that $W(p)$ can change sign only where it is a decreasing function of pressure, i.e., increasing upward (since for $W=0, d W / d p$ is negative). Thus, if $W$ is nonnegative at the cloud base it cannot become negative along the parcel's entire path upward. This argument does not hold for the downward trajectory, but as long as the motion there is maintained by negative buoyancy, the ESLWC must remain positive. This follows from the expression for buoyancy

$$
B=g\left(\frac{T-T_{0}}{T_{0}}-V\right)+S
$$

where $B$ is the buoyancy, $g$ the gravity acceleration, and $S$ the correction for the virtual temperature effect, which is almost always positive. Comparing Eqs. (3) and (1), and noting that $L / c_{p} \approx 2500 \mathrm{~K}$ is much greater than $T_{0}$, one can easily see how negative buoyancy yields a positive ESLWC. (Exceptions occur only in rare situations involving negative $S$.)

Theorem 2 follows from the integration of Eq. (2), when pressure $p$ is expressed as a function of time $t$. Then

$$
W(t)=W^{*}+\int_{t^{*}}^{t}\left(\gamma-\Gamma_{d}\right) \frac{d p}{d t} d t-\int_{t^{*}}^{t} \mu \frac{d p}{d t} W d t
$$

where the asterisk refers to the initial conditions. If $\Gamma_{d}$ is independent of $\mu$, the two first terms of the right side of Eq. (4) can be identified as the adiabatic ESLWC. A correction to this value due to entrainment is given by the last integral. This correction cannot be positive since $\mu(d p / d t)=d \ln M / d t$ is nonnegative according to the defiintion of entrainment, and $W \geqslant 0$ according to Theorem 1; hence, Theorem 2 is proved.

The model of a single, uniform parcel (or the formally similar model of a steady jet) which entrains air only from a cloudless environment, although commonly used, may appear too crude in some applications. In particular, there might be doubts whether conclusions derived from the use of such a model could be applied to local conditions within the cloud. More appropriate here is a model of a parcel which entrains the air not only from the environment but also from other cloud parcels with varying thermodynamical properties. We shall thus generalize our results to such a model, assuming for the sake of mathematical simplicity that at each pressure $p$ the parcel entrains air from a finite number of sources, i.e., from other cloud parcels and from a cloudless environment.

For such a model, Eq. (2) can be generalized as follows (see Appendix):

$$
\frac{d W}{d p}=\frac{c_{p}}{L}\left(\gamma-\Gamma_{d}\right)-\mu W+\sum_{k} \mu_{k} W_{k}
$$

where $k$ is the index used for labeling various sources of entrained air, $W_{k}$ is the ESLWC of the $k$ th source, and $\mu_{k}$ (the partial coefficient of entrainment) $=(1 / M)(d M / d p)_{k}$. Here $(d M / d p)_{k}$ is the rate of entrainment from the $k$ th source, and $\mu$ (the total coefficient of entrainment $)=(1 / M)(d M / d p)=d \ln M / d p$ $=\sum_{k} \mu_{k}$. The environmental ESLWC is defined equal to zero, in accordance with Eq. (1). Eqs. (2) and (5) are also valid for unsaturated parcels for which $V=0$.

For the model under consideration, the following analogues of Theorems 1 and 2 are valid:

Theorem 3. If $\gamma<\Gamma_{d}$ above the cloud base, the ESLWC at the cloud base is nonnegative for the whole lifetime of the cloud, and nonbuoyant downdrafts (as well as entrainment from them) are not considered, then the ESLWC of any cloud parcel must be nonnegative.

THEOREM 4. If (in addition to the assumptions of Theorem 3) $\gamma, \Gamma_{d}$, and the cloud base conditions can be assumed to be time-independent and identical for all cloud parcels, then the ESLWC of any cloud parcel cannot surpass the adiabatic value (which, under such assumptions, is a function of pressure, and is identical for all cloud parcels).

For parcels moving downward under negative buoyancy, Theorem 3 can be demonstrated in the same way as Theorem 1; with respect to parcels moving upward, the supposition that Theorem 3 does not hold leads to a contradiction, since no such parcel can be the first in which negative ESLWC appears. Indeed, negative ESLWC can appear only under the condition that $\sum_{k} \mu_{k} W_{k}>0$, since otherwise Theorem 3 must follow from Eq. (5) in the same way that Theorem 1 follows from Eq. (2). However, this condition means that at least for some $k, W_{k}<0$ (since for upward motions all $\mu_{k}<0$ ); i.e., negative ESLWC is already present in the cloud. Thus, negative ESLWC cannot appear in the cloud unless some of the conditions of validity of Theorem 3 are violated.

Theorem 4 can be proved similarly by demonstrating that no parcel can be the first one in which a superadiabatic ESLWC appears. For this purpose let us express pressure $p$ as a function of time $t$ and rewrite 
(5) as

$$
\begin{aligned}
\frac{d W}{d t}=\left(\gamma-\Gamma_{d}\right) \frac{d p}{d t}+\sum_{k} \mu_{k} \frac{d p}{d t} & \left(W_{k}-W\right) \\
& =\frac{d W_{a}}{d t}+\sum_{k} \mu_{k} \frac{d p}{d t}\left(W_{k}-W\right),
\end{aligned}
$$

where $W_{a}$ is the adiabatic ESLWC.

From Eq. (6) it follows that for the ESLWC in a parcel to become superadiabatic the last term of Eq. (6) must be positive at the moment when adiabatic ESLWC is being surpassed, i.e., when $W=W_{a}\left[\right.$ since $\mu_{k}(d p / d t)$ $\geqslant 0$, by definition of entrainment], i.e., superadiabatic ESLWC must already be present in other cloud parcels.

Let us notice that by applying the term "adiabatic" in Theorem 4 to the greatest adiabatic ESLWC which for a given range of cloud base conditions can occur at a given pressure, we can avoid the requirements of uniformity and time independence of the cloud base conditions, which sometimes may not be fulfilled with sufficient accuracy.

Theorems 3 and 4 are also valid for more general models in which a parcel can interact with a continuous family of other parcels. Generalization of the proofs requires only that the sums in Eqs. (5) and (6) be replaced by integrals, which can be done easily under the usual formal restrictions.

\section{Some applications of the notion of ESLWC}

The simplicity of Eqs. (2) and (5) makes the ESLWC a convenient parameter for describing and analyzing some of the thermodynamical processes in clouds and for expressing other physical quantities, particularly in one-dimensional models of convective clouds. Applications of the ESLWC may also be useful in the analysis of cloud physics measurement data. Three such applications are described below.

\section{a. Determination of the bulk entrainment coefficient}

The integration of $\mathrm{Eq}$. (2), using pressures $p_{1}$ and $p_{2}$, with the assumption that $\gamma-\Gamma_{d}$ and $\mu$ are constant, yields

$$
\begin{aligned}
W\left(p_{2}\right)=W\left(p_{1}\right) \exp \left[-\mu\left(p_{2}-p_{1}\right)\right] & \\
+\left(\gamma-\Gamma_{d}\right) & \frac{1-\exp \left[-\mu\left(p_{2}-p_{1}\right)\right]}{\mu} .
\end{aligned}
$$

When $W\left(p_{1}\right)=0, \mathrm{Eq} .(7)$ yields a particularly simple form; in practice, such $p_{1}$ can be assumed to coincide with the cloud base level.

Numerical solution of Eq. (7) can be used conveniently for determination of $\mu$ (which in this case represents the bulk, average entrainment between $p_{1}$ and $\left.p_{2}\right)$, provided that $\gamma, W\left(p_{1}\right)$ and $W\left(p_{2}\right)$ are known from measurements. With respect to the classical approach of Stommel (1947), this method has the advantage of being independent of the environmental humidity stratification, which is less uniform horizontally than the temperature stratification, so that standard radiosonde data may not appear representative for the direct vicinity of the cloud.

Whether the formula in Eq. (7) gives $\mu$ more accurately than the classical one depends on the particular details of stratification and on the type and accuracy of the measurements available, but, in any case, comparison of the results given by these two methods might be interesting.

\section{b. Detection of liquid water exchange}

Eq. (5) and Theorems 3 and 4 are based upon a model seemingly general enough to be applied to local conditions within real clouds. Thus, if negative or superadiabatic values of the ESLWC are observed somewhere in the cloud, some violations of the conditions for validity of Eq. (5) or the theorems following from it must be occurring there. The only processes in which such violations take place and which might be expected in typical convective clouds are nonbuoyant downdrafts (and eventually entrainment from them to other cloud parcels) and/or exchange of liquid water between cloud parcels in the form of precipitation. In particular, measurements that indicate negative ESLWC values in cloud regions that can scarcely be affected by nonbuoyant downdrafts also indicate a net loss of water by precipitation. On the other hand, superadiabatic values of the ESLWC indicate the so-called process of accumulation. Of course, gains or losses of liquid water too small for making ESLWC superadiabatic or negative cannot be detected by this method, but the ESLWC is nevertheless a more sensitive detector of such processes than the ordinary liquid water content.

It is worth noting that since at the cloud base the ESLWC can be assumed to be zero, the adiabatic ESLWC can be given by

$$
W_{a}=\frac{c_{p}}{L} \int_{p_{\mathrm{bag}}}^{p}\left(\gamma-\Gamma_{a}\right) d p
$$

and for practical purposes can be found easily from a typical aerological diagram as being proportional to the temperature difference between the stratification curve and the dry adiabat which intersects the former at the cloud base level.

\section{c. Estimation of liquid water content from temperature data}

In the regions of clouds where the effects of both nonbuoyant downdrafts and precipitation exchange can be excluded, e.g., in the cores of young cumulus clouds, 
Theorems 3 and 4 yield the inequalities

$$
\frac{c_{p}}{L}\left(T-T_{0}\right) \leqslant V \leqslant W_{a}+\frac{c_{p}}{L}\left(T-T_{0}\right),
$$

which can be used for estimation of $V$ by means of the temperature data alone. As follows from Eqs. (8) and (9), this estimation is particularly good for relatively thin layers with little stability, where $W_{a}$ is small.

\section{APPENDIX}

\section{Derivation of Eqs. (2) and (5)}

Retaining all symbols introduced in the main text, let us consider a thermodynamic process in which a parcel of humid air with variable mass $M$ changes its pressure and undergoes isobaric mixing with the unsaturated environment (all environmental parameters will henceforth be labeled with subscript 0 ). The principle of conservation of any intensive parameter $S$ with specific density $s$ can be expressed by

$$
\frac{d S}{d p}=\frac{d M s}{d p}=M P+s_{0} \frac{d M}{d p},
$$

where $P$ denotes production of $S$ per unit mass and pressure. Eq. (A1) yields

$$
\frac{d s}{d p}=P+\mu\left(s_{0}-s\right) .
$$

Designating $q$ as the specific humidity and $Q$ its value for saturated air, and then applying (A2) to the total specific water content of a saturated parcel which undergoes mixing with an unsaturated environment $\left(V_{0} \equiv 0\right)$, we obtain

$$
\frac{d V}{d p}=-\mu V-\mu\left(Q-q_{0}\right)-\frac{d Q}{\partial T} \frac{d T}{d p}-\frac{\partial Q}{\partial p} .
$$

Now, let us apply (A2) to the simplified expression for enthalpy of humid air, given by the expression

$$
h=c_{p} T+L q .
$$

Recalling that in this case $P=v$, and assuming that $L$ and $c_{p}$ are constant, we get

$$
\frac{d T}{d p}=\frac{\frac{v}{c_{p}}-\frac{L}{c_{p}} \frac{\partial Q}{\partial p}-\mu\left[\left(T-T_{0}\right)+\frac{L}{c_{p}}\left(Q-q_{0}\right)\right]}{1+\frac{L}{c_{p} \partial Q} \frac{\partial Q}{\partial T}}
$$

or

$$
\frac{d T}{d p}=\Gamma_{w}-\mu \frac{\left(T-T_{0}\right)+\frac{L}{c_{p}}\left(Q-q_{0}\right)}{1+\frac{L}{c_{p}} \frac{\partial Q}{\partial T}},
$$

where $\Gamma_{w}$ is the moist-adiabatic lapse rate with respect to pressure.

Several variants of Eqs. (A3) and (A5) or (A6) are commonly used in one-dimensional cloud models. In the case of the unsimplified expression Eq. (A4), in which $L$ and $c_{p}$ are variable and the enthalpy of liquid water is also included, we obtain after rather cumbersome transformations, an equation which differs from Eq. (A5) only by a factor $c_{p 0} / c_{p}$ with the term $\left(T-T_{0}\right)$. Since this factor generally differs from unity by less than $1 \%$, the approximation Eq. (A5) is, in most cases, fully satisfactory.

Recalling the identity

$$
\Gamma_{w}=\Gamma_{d}-\left(\frac{\partial Q}{\partial T} \Gamma_{w}+\frac{\partial Q}{\partial p}\right)_{c_{p}}^{L}
$$

which follows directly from the definition of $\Gamma_{w}$, and substituting $d T / d p$ from (A6) into (A3), we get

$$
\begin{aligned}
\frac{d V}{d p}= & \frac{c_{p}}{L}\left(\Gamma_{w}-\Gamma_{d}\right) \\
& +\mu \frac{\partial Q}{\partial T} \frac{\left(T-T_{0}\right)+\frac{L}{c_{p}}\left(Q-q_{0}\right)}{1+\frac{L}{c_{p}} \frac{\partial Q}{\partial T}}-\mu V-\mu\left(Q-q_{0}\right) .
\end{aligned}
$$

Subtracting $\gamma=d T_{0} / d p$ from both sides of Eq. (A6), we have

$$
\frac{d}{d p}\left(T-T_{0}\right)=\left(\Gamma_{w}-\gamma\right)-\mu \frac{\left(T-T_{0}\right)+\frac{L}{c_{p}}\left(Q-q_{0}\right)}{1+\frac{L}{c_{p}} \frac{\partial Q}{\partial T}} .
$$

Dividing Eq. (A9) by $L / c_{p}$, subtracting the results from Eq. (A8), and performing some simple algebraic transformations, we finally obtain Eq. (2). The only essential simplification made at this latest stage of the derivation is the assumption of constant $L / c_{p}$. Without this assumption, the term $\left(T-T_{0}\right) d\left(c_{p} / L\right) / d p$ would have appeared on the right side of Eq. (2). It is easy to verify that in most cases of practical interest this term is two orders of magnitude smaller than the other components of (2) and can be safely ignored.

Eq. (5) can be derived in a similar way. For this purpose let us note that for a parcel which isobarically entrains air from various sources (labeled by subscript $k$ ) the analogue of (A1) is

$$
\frac{d s}{d p}=P+\sum_{k} \mu_{k}\left(s_{k}-s\right) .
$$

Applying (A10) to the total water content and enthalpy of the saturated parcel, and using (A7), we obtain the 
following analogues to Eqs. (A8) and (A9):

$\frac{d V}{d p}=\frac{c_{p}}{L}\left(\Gamma_{w}-\Gamma_{d}\right)+\sum_{k} \mu_{k}\left[\frac{\partial Q}{\partial T} \frac{\left(T-T_{k}\right)+\frac{L}{c_{p}}\left(Q-q_{k}\right)}{1+\frac{L}{c_{p}} \frac{\partial Q}{\partial T}}\right.$ $\left.-\left(V-V_{k}\right)-\left(Q-q_{k}\right)\right\}$

$\frac{d}{d p}\left(T-T_{0}\right)=\Gamma_{w}-\gamma-\sum_{k} \mu_{k}\left(\frac{\left(T-T_{k}\right)-\frac{L}{c_{p}}\left(Q-q_{k}\right)}{1+\frac{L}{c_{p}} \frac{\partial Q}{\partial T}}\right\}$.

Multiplying (A12) by $c_{p} / L$ and subtracting it from (A11), we obtain

$$
\frac{d W}{d p}=\frac{c_{p}}{L}\left(\gamma-\Gamma_{d}\right)
$$

$$
-\sum_{k} \mu_{k}\left[V-\frac{c_{p}}{L}\left(T-T_{k}\right)-V_{k}\right]
$$

which by means of the identity

$$
T-T_{k} \equiv\left(T-T_{0}\right)-\left(T_{k}-T_{0}\right)
$$

can be transformed

$$
\begin{aligned}
& \frac{d W}{d p}=\frac{c_{p}}{L}\left(\gamma-\Gamma_{d}\right)- {\left[V-\frac{c_{p}}{L}\left(T-T_{0}\right)\right] \sum_{k} \mu_{k} } \\
&+\sum_{k} \mu_{k}\left[V_{k}-\frac{c_{p}}{L}\left(T_{k}-T_{0}\right)\right] \\
&=\frac{c_{p}}{L}\left(\gamma-\Gamma_{d}\right)-\mu W+\sum_{k} \mu_{k} W_{k},
\end{aligned}
$$

i.e., to $\mathrm{Eq}$. (5).

Let us note that within the accuracy accepted in this paper, specific humidities and liquid water contents might be replaced by the corresponding mixing ratios.

Acknowledgments. The author is indebted to the National Hail Research Experiment at NCAR and personally to Drs. W. C. Swinbank, T. Kyle and J. Rosinski for their kind assistance in preparation of the final text of the paper.

\section{REFERENCES}

Stommel, H., 1947: Entrainment of air into a cumulus cloud. J. Meteor., 4, 91-94. 